Revista Iberoamericana, Vol. LXXVIII, Núms. 238-239, Enero-Junio 2012, 259-275

\title{
TOMO LO QUE ENCUENTRO: SOBRE OSVALDO LAMBORGHINI Y LA CIENCIA-FICCIÓN
}

\author{
POR \\ Santiago Deymonnaz \\ Universidad Carlos III, Madrid
}

ESCRIBIR UNA NOVELA

\begin{abstract}
Escribir una novela en las últimas décadas de este siglo, aunque ya surgió una complicación que nos deja perplejos. Algunos sabios de EE.UU. y la Unión Soviética, apoyados en la teoría de la relatividad, ya hablan de un "pliegue” matemático -no se exciten, ni me pregunten acerca del "pliegue” porque yo no podría explicarlo, aunque no me negaría a aproximar a quien le interese una bibliografía seria. Distraídos, o “plegados” por el tema bibliográfico, olvidamos lo principal: algunos sabios niegan que haya existido un siglo xx. [...]

Escribir una novela.

Que todo un siglo desapareciera, matemáticamente plegado. (Lamborghini 118)
\end{abstract}

Aunque reacia a una simple inscripción en géneros consagrados o formas establecidas, la escritura de Osvaldo Lamborghini (1940-1985) nos reenvía insistentemente hacia la institución literaria. Es verdad que este reenvío es condición de toda literatura $-\mathrm{y}$ por lo tanto siempre estará presente, en mayor o menor medida, en el más autosuficiente de los textos-, pero también es verdad que en el caso de Lamborghini adquiere un rol predominante. Se trata de un reenvío obstinado y de gran trascendencia: se encarga de establecer sus propias genealogías. Si el destino de su escritura, como sugiere Nicolás Rosa, es un destino incierto -sus textos no pueden formar escuela ni fundar estilos-, sus orígenes no son ciertamente tan oscuros (125). Con una porfía notable, los textos de Lamborghini dan por sentada la literatura, la suponen, y esta porfía es tal que hace posible y tentador encontrar los hilos con los que se teje su escritura.

Como ya la crítica lo ha señalado, estos hilos conducen principalmente a la propia literatura argentina donde su obra irrumpe. No obstante, el juego con el pasado -con la literatura pasada- no se limita a una tradición. Convoca también formas y géneros que la exceden -aunque fatal y borgeanamente estos sean luego reconducidos a la misma escena. Una de estas formas puede ser la ciencia-ficción (CF), que emerge difusa en 
una de las últimas y más extensas obras de Lamborghini -Tadeys-, escrita hacia 1983 y publicada de manera póstuma diez años más tarde. Ricardo Strafacce, en su vasta biografía del autor, señala que en la primera parte de Tadeys se alternan un relato de iniciación con un relato de CF (779). Un estudioso de la obra de Lamborghini sabrá que esta doble evocación de géneros altamente codificados no debe tomarse al pie de la letra, lo cual no implica que haya que descartarla sin más. Si bien es difícil clasificar la obra de Lamborghini, autor excéntrico, no por ello debemos abstenernos de estudiar los usos del género -en nuestro caso, la CF- que lleva a cabo su escritura. Ya en las primeras páginas podemos constatar que Tadeys no es sensu estricto un relato de iniciación ni un relato de CF, y sin embargo, como señala el biógrafo, ambos géneros están allí, de alguna manera implicados.

Nuestro escrito pretende precisamente leer la "novela" Tadeys a la luz de ciertas claves de la CF, especialmente en la modalidad que la crítica ha vinculado a las obras producidas en América Latina. La lectura esconde, en realidad, la operación inversa: leer estas claves a la luz de Tadeys. Se trata por tanto de ver cómo funciona la CF en el interior de la obra, pero también de pensar qué nos puede decir este uso del género sobre el género mismo o sobre la literatura en general. Ahora bien, al acercar estos dos universos, estos dos sistemas que son, por un lado, la obra de Osvaldo Lamborghini y, por el otro, la litertura de CF, nos hemos enfrentado desde el primer momento con dos grandes complicaciones. Por un lado, la ya mencionada dificultad -algunos críticos dirán la imposibilidad- de clasificar una obra como Tadeys. En segundo término, la dificultad -otros críticos dirán también la imposibilidad- de definir un género como la CF.

En lo que atañe a la primera, a la imposibilidad de clasificar una obra como Tadeys, debemos precisar que no se trata ya de que el texto no pueda adscribirse a la CF ni a ningún otro género narrativo establecido; con Tadeys el mismo concepto de "obra" parece tornarse inoportuno. Texto fragmentario, escrito en cuadernos y apuntes sueltos, su forma final no la debemos al autor, sino a su albacea literario, quien presenta el texto como una "novela". ¿Pero estamos realmente ante una novela? Frente a esta pregunta, Graciela Montaldo propone que la idea de "obra” que subyace en Tadeys no apunta a ninguna forma fija, sino al mismo acto de escribir. Los personajes aparecen y desaparecen, dejando sus biografías inacabadas. La narración realiza saltos temporales hacia atrás y hacia delante de manera "arbitraria". Las anécdotas se interrumpen. La historia que allí se narra no tiene comienzo ni conclusión, sino que se expande y prosigue. Esto no podía ser de otra manera, sugiere Montaldo, dadas las pretensiones de la obra: escribir una novela total, fabular con ella la historia del poder y de la representación -poder y representación: dos de los posibles temas de Tadeys. A fines del siglo xx -sigue Montaldo- esta historia no podía ser escrita, ni siquiera como novela. Debía, por tanto, permanecer en los fragmentos de esas carpetas intervenidas (256-76). Y es entre estos "fragmentos” que aparecerán los elementos que

Revista Iberoamericana, Vol. LXXVIII, Núms. 238-239, Enero-Junio 2012, 259-275
ISSN 2154-4794 (Electrónico) 
nos reenvíen al universo de la CF. Pero -como ya lo hemos sugerido- este reenvío no tendrá el espesor de una inscripción, ni siquiera el de una parodia. La CF aparecerá más bien con la consistencia de un eco, como los fragmentos que flotan en el espacio literario después de la explosión de la literatura que opera su obra.

La segunda dificultad la encontramos del lado del género. Si nos disponemos a leer Tadeys a partir de algún tipo de vínculo con la CF, debemos antes precisar qué entendemos por ella. ¿Qué es, en definitiva, la CF? ¿Cómo definimos o limitamos ese cuerpo, extraño a la obra de Lamborghini, pero que participa en ella, que se intercala allí con otros cuerpos? Entre los críticos del género es proverbial el atolladero en el que se han visto metidos aquellos que han intentado ofrecer una definición precisa de la CF. Es como si el género, en su cercanía o superposición con géneros lindantes -la fantasía, la utopía, etc.-, revelara esa ley de impureza o principio de contaminación que Jacques Derrida propusiera como condición de posibilidad de todo género (57). Por ello, no tomaremos aquí la CF como un rótulo que se pueda asignar a determinadas obras y negar a otras, así como no pretenderemos responder a la pregunta de si es posible establecer límites precisos para este género. En la medida en que no intentaremos argumentar la consideración de Tadeys como una obra de CF, la cuestión del límite no será determinante. Antes, adoptaremos una posición como la propuesta por Carl Freedman, según la cual la CF -o cualquier otro género literario- debería ser concebida como un elemento o como una tendencia que opera, de manera más o menos activa, dentro de determinados textos, entendidos estos como estructuras complejas (181-82). De acuerdo con esto, no habría ningún texto que fuera la encarnación pura y perfecta de la CF, así como no habría prácticamente ningún texto en el que no estuviera funcionando alguna de las tendencias del género (más adelante intentaremos describir a grandes rasgos estas tendencias, para determinar los modos en que éstas podrían aparecer en la escritura de Lamborghini).

Nos encontramos, entonces, con un texto fragmentario por un lado y con un género poroso por el otro. El encuentro de ambos no resultará en un argumento cerrado. Ya lo hemos dicho: no se trata de leer la obra de Lamborghini como relato de CF. Tampoco se trata de presentar a Osvaldo Lamborghini como lector del género. Si aparecen en Tadeys ciertas marcas que nos reenvían a la CF, éstas no revelan más que un juego: el juego con la literatura que es central en la obra de este escritor y en la consistencia de su intervención en la escena literaria. En el fondo, lo que intentamos leer es sólo una secuencia de una operación literaria más vasta.

¿QUÉ O QUIÉN ERA ESO?

¿Qué o quién era eso?, se preguntó Maker. “Un hombre en estado salvaje”, se dijo. No, pues el ser que había aparecido, cuyo cuerpo era semejante al de un niño de trece años, tenía la cara (horrible, abominable casi de tan fea) completamente arrugada,

\footnotetext{
Revista Iberoamericana, Vol. LXXVIII, Núms. 238-239, Enero-Junio 2012, 259-275 ISSN 0034-9631 (Impreso) 
aureolada, para su propia -Maker- irrisión, de un círculo monjil de pelo albino. Sólo allí tenía pelo. Como si se depilara (absurdo), ni uno solo más se veía en el resto del cuerpo. Pensó: "Un mono lampiño", y se sintió completamente estúpido: "Mono lampiño" era una contradicción en los términos, como "pelado con trenzas”, que a tantos les costó la vida (en la hoguera) porque se refería, para ponerla en ridículo, a la Orden del Obispo [...] (Lamborghini 187)

La cita da cuenta del primer encuentro con un tadey, de ese maravilloso descubrimiento que hace el Padre Maker en su destierro por las montañas del norte, una zona desconocida y desértica de La Comarca. ${ }^{1}$ En esta escena y en todo el relato, el tadey se presenta como una raza nueva, ambigua, a mitad de camino entre el hombre y el animal, "un animal que en cuanto a parecido con el hombre derrotaba a los impúdicos monos” (Lamborghini 188). Se trata de una raza llamada a transformar la fisonomía del país, sus relaciones sociales, su política, su economía, sus costumbres sexuales y hasta su posición en el concierto de las naciones. ${ }^{2}$

Esta singularidad del territorio inventado, la existencia y la centralidad de esta "nueva raza", acerca la obra, Tadeys, a ciertos textos considerados fundadores de la $\mathrm{CF}$, textos que podrían colocarse en la prehistoria o en los albores del género. Pienso principalmente en Gulliver's Travels (1726), pero también en la larga serie de textos que, mediante la introducción de una nueva especie, concibieron un mundo "parecido" al nuestro -una tradición literaria que por cierto no carece de antecedentes en la literatura argentina: los trogloditas de "El inmortal" y los yahoos de "El informe de Brodie" son dos ejemplos centrales en esta literatura. ${ }^{3}$

Por tanto, la misma existencia de estos mamíferos que dan título a la obra se convierte, en mi lectura, en el primer $-\mathrm{y}$ tal vez principal- elemento activo que

1 La Comarca o LacOmar -el topónimo, como tantos otros nombres en el relato, es inestable-es el territorio fantástico que concibe el texto de Lamborghini. Allí es donde transcurre la acción de esta “novela”.

2 Con el tiempo, la crianza y la industrialización del tadey "gracias a las generaciones que se dedicaron a la cruza y perfeccionamiento de este mamífero” llevaron a La Comarca a una posición privilegiada “en lo militar, la expansión científica y el nivel alimenticio que ocupamos frente a otras naciones” (Lamborghini 69).

3 Estos dos relatos de Jorge Luis Borges (1899-1986) - a los que se podría añadir “Los donguis”, de Juan Rodolfo Wilcock (1919-1978)- son algunos de los antepasados nacionales de los tadeys. Incluso se podría decir que el descubrimiento que hace el Padre Maker en el texto de Lamborghini de esta singular raza y de sus intensas prácticas sexuales no son otra cosa que aquello que el narrador de "El informe de Brodie” omite pudorosamente: “Traduciré fielmente el informe, compuesto en un inglés incoloro, sin permitirme otras omisiones que las de algún versículo de la Biblia y la de un curioso pasaje sobre las prácticas sexuales de los Yahoos que el buen presbiteriano confió pudorosamente al latín” (Borges 140). La narración del encuentro del Padre Maker con los tadeys se centra precisamente en la curiosa sexualidad de estos seres. Sobre la participación de este motivo -una raza nueva, a mitad de camino entre el hombre y el animal- en la obra de Borges y sobre la relación de su obra con la CF, puede consultarse Borges y la ciencia ficción de Carlos Abraham.

Revista Iberoamericana, Vol. LXXVIII, Núms. 238-239, Enero-Junio 2012, 259-275
ISSN 2154-4794 (Electrónico) 
coloca el texto en la órbita de la CF. Dicho de otra manera y atendiendo a la tradición crítica sobre el género, los tadeys vendrían a ocupar el lugar de ese novum que Darko Suvin concibiera como el eje teórico de la CF en su ya clásico intento por describir esta literatura. Los tadeys $-\mathrm{y}$ con ellos todo el territorio que configuran con su omnipresencia- constituyen esa escena, ese locus espacio-temporal desconocido. ${ }^{4}$ Son ellos, los tadeys, el centro de la ficción narrativa, si bien son otros -todos humanos- los protagonistas del relato: el Padre Maker, Seer Tijuán, el doctor Ky, Jones Hien, Dam Vomir, etc. Y son el centro de la ficción porque son ellos los que determinan el tiempo y el espacio, la totalidad social y las relaciones humanas de La Comarca, y porque son ellos, los tadeys, los que producen el primer extrañamiento en el lector, condiciones -todas estas- de la innovación o el novum formulado por el crítico yugoslavo ("The State of the Art” 38-39).

El modo en que se nos revela esta raza y el mundo por ella condicionado nos conduce a otra tendencia propia de la CF. En el artículo citado anteriormente, Suvin reconoce como una estrategia común en esta narrativa el uso de ciertas formas de la novela de aprendizaje como medio para que el protagonista comprenda -y con él, el lector- el novum que caracteriza el mundo del relato (39). En Tadeys, es precisamente a través del crecimiento y las experiencias del niño Seer Tijuán que tenemos el primer contacto con La Comarca, con la estructura socio-política, la legislación y los hábitos sexuales de este territorio. A esta participación en el relato de la novela de aprendizaje (779), ya señalada por Strafacce, se puede sumar la narración del descubrimiento que lleva a cabo el Padre Maker: su destierro y encuentro con los tadeys en las montañas del norte constituyen una verdadera experiencia de iniciación y, si bien esta anécdota se nos refiere al final del texto, es este encuentro el que nos revela la naturaleza de la nueva raza.

\section{El Estado Espejo y su Orden Sexual}

Lástima que al margen de este diario que llevo, que intento llevar, y me ha servido ya para el prólogo de mi libro “El Estado Espejo y su Orden Sexual”, igual escucho: aullidos, dolor. (Lamborghini 98)

4 En un artículo de 1979, Suvin planteaba en estos términos su noción de novum: "How can the domain of CF be determined, on what does it hinge as its theoretical axis? I do not see any tenable internal determination of CF which would not hinge on the category of the novum. A novum or cognitive innovation is an important difference superadded to or infused into the author's empirically 'known' -i.e., culturally defined-world, or, more usefully, it is an important deviation from the author's norm of reality. [...] The postulated innovation may run from the minimum of one discrete new 'invention' (gadget, technique, relationship) to the maximum of a scene (spatiotemporal locus), agent (main characters), and/ or relations basically new and unknown in the author's and the implied reader's environment" "The State of the Art” 36).

\footnotetext{
Revista Iberoamericana, Vol. LXXVIII, Núms. 238-239, Enero-Junio 2012, 259-275 ISSN 0034-9631 (Impreso) ISSN 2154-4794 (Electrónico)
} 
Las palabras pertenecen a la pluma del doctor Emorebe Ky, y el diario al que hacen referencia es el que escribe a bordo del buque-cárcel de amujerar, el proyecto insignia de La Comarca; los aullidos y el dolor son una parte necesaria del proyecto. Este barco, un antiguo transatlántico en desuso atracado a unas cuantas millas de la costa, es el escenario donde el saber psiquiátrico del doctor Ky y la autoridad tiránica de Jones Hien llevan a cabo el llamado "Método para dulcificar las costumbres de adolescentes violentos" (Lamborghini 75). ${ }^{5}$

El método, un completo programa eugenésico, no es otra cosa que la puesta en práctica del modelo de Estado que podríamos leer en el libro del doctor Ky, y representa una miniatura del sistema político de La Comarca. En muchos aspectos, la inclusión de este proyecto es fundamental en la obra. Si los tadeys, centro de la ficción, modificaban la fisonomía del territorio, el buque-cárcel de amujerar se convierte en su forma más acabada y perfecta, el espacio utópico de La Comarca.

Al leer con atención las reflexiones que el doctor Ky consigna en su diario podemos concluir, junto con la crítica, que Tadeys comporta toda una teoría o -por lo menos- una lectura del Estado moderno. En definitiva, más que ante una novela, estaríamos, como sugiere Montaldo, ante una fábula: una fábula sexual y política construida a partir de una serie de fragmentos sobre el poder y la autoridad (265). Así, todas las aventuras que se suceden en la obra pueden leerse en la clave de una literatura convertida en teoría política: empezando por el objetivo del doctor Ky de "abismar al individuo", por los tadeys -seres a mitad de camino entre el hombre y el animal, "abismados" por naturaleza- y por el propio lugar asigando a esta nueva raza en La Comarca -alimento y cuerpo sexual. ${ }^{6}$

5 "El proyecto consistía en lo siguiente. Como a todo el mundo, a La Comarca llegó la moda del joven navajero y violento. Al principio se optó por la vía tradicional, el reformatorio. Pero el genio de dos geniales ambiciosos, hiena Jones y araña Ky, concibió otro método. [...] En cuanto subían a bordo, los convictos eran entregados a bufas especialistas en transformar en damas a los que se creían 'demasiado' viriles. En cuanto subían a bordo los sodomizaban. Así, constantemente, la primera semana. Perdían hasta el nombre. Para llamarlos decían: 'Venga para acá, puto 14', o cosas por el estilo. Después, en apariencia, el tratamiento se dulcificaba. Venía la enseñanza de modales femeninos y el complicado aprendizaje de comportarse como lo hacían las señoras” (Lamborghini 76).

6 Lo que se propone Ky, junto con el aparato estatal de La Comarca, es alcanzar en el individuo el mayor grado de absorción del modelo, de hacerlo desaparecer bajo el peso del Estado: "el Estado, absoluto espejo, y el individuo que en él se sumerge, también absoluto, absolutamente, aunque no tiene ‘construcción’ y por lo tanto muere”, escribe Ky (Lamborghini 99). Como propone Fermín Rodríguez, se postula en Tadeys una relación entre el individuo y el Estado a través del cuerpo: se presenta un yo que "ya no es tuyo", un yo que "es forzado a abandonar sus lugares de identificación por un poder de hacer y rehacer los lazos de identidad en torno a puntos de dolor y de goce que atan la vida de un cuerpo a un modelo" (177). En este sentido, saber y poder van de la mano: la autoridad tiránica de Dam (joven y todopoderoso alcalde de La Comarca) "trabaja sobre el mismo umbral de actualización que el saber psiquiátrico del doctor Ky" (177). Este mecanismo, que opera en la construcción de la identidad del individuo, recorre todo el texto e incluso lo desborda, apareciendo en otros escritos de Lamborghini. Se trata de un determinismo -la

Revista Iberoamericana, Vol. LXXVIII, Núms. 238-239, Enero-Junio 2012, 259-275
ISSN 2154-4794 (Electrónico) 
Como señalábamos al comienzo de este artículo, en Tadeys los fragmentos se suceden, interrumpiéndose entre ellos, abriendo líneas argumentales que quedan en su mayoría truncas. En esta proliferación, la fábula se complejiza. Sin embargo, algo parece dirigir la obra: el espacio ficcional de La Comarca. No es aventurado suponer que cada fragmento está allí, en última instancia, para contribuir a la creación y el diseño de este territorio imaginario, para definir la arquitectura de ese Estado Espejo y su particular Orden Sexual. Se podría decir que La Comarca, mediante las aventuras de sus personajes, aparece retratada en sus aspectos sociopolíticos más relevantes. Su geografía, su religión, su sistema de gobierno, su economía, su arte de la guerra, etc., todas estas esferas ocupan un papel decisivo en el avance de la escritura. En este sentido, en este dar cuenta de un territorio convertido en motor narrativo, la fábula se convierte en ficción utópica; y es precisamente en la utopía donde volvemos a encontrar otro de los elementos propios de la CF.?

La utopía, entendida como discurso, con sus características y sus leyes, ha estado siempre vinculada con la CF. La tarea del escritor de este género es en en buena medida análoga a la del escritor utópico. El narrador argentino Eduardo Abel Giménez (1954-), en una antología crítica del género, describía esta tarea en los siguientes términos: "Quien se sienta a escribir un relato de ciencia ficción debe detenerse a describir el mundo (el punto de la recta) donde los lectores habrán de situarse. Esta tarea a veces es agradable, y a veces un lastre que no hay más remedio que soportar" (Giménez 155). Lastre o motor de la escritura -en el caso de la obra de Lamborghini nos inclinamos por la segunda de estas dos opciones-, esta tarea de describir un nuevo escenario acerca ambos géneros. En esta línea, podríamos decir que la CF sólo se puede escribir en un horizonte utópico. ${ }^{8}$

El propio Darko Suvin ha estudiado esta relación. En "Science Fiction and Utopian Fiction: Degrees of Kinship” no sólo señala la utopía como una de las raíces históricas del género, sino que llega incluso a proponerla como una de sus formas,

violación convierte a los niños o jóvenes que la padecen, sino en "mujeres" por lo menos en "vulgares putos”- que está presente también en obras como El Pibe Barulo (Strafacce 779).

7 Sobre las características de la ficción utópica, se puede consultar el todavía vigente Sade, Fourier, Loyola de Roland Barthes. Allí, el crítico francés destaca en la obra de esos tres autores una serie de elementos que servirían para caracterizar ciertas tendencias del discurso utópico y que, por cierto, estarían presentes en Tadeys: la importancia del detalle, la estructura fragmentada y móvil -intermitente- de la escritura, la preponderancia en el lenguaje de las relaciones de insistencia -que prescinden de un centro-sobre las de consistencia, etc.

8 Atendiendo al sentido estricto de los términos griegos que componen la palabra, no considero aquí la utopía como un lugar ideal u optimista, sino más bien como un no-lugar o un lugar-otro. En los límites de este trabajo, la utopía es considerada como un lugar perfecto sólo en la medida en que esta perfección no se encuentra atravesada por ninguna consideración moral. La utopía -La Comarca, en definitiva- es un lugar "perfecto" así como podríamos decir que la suma $2+2=4$ es una operación "perfecta”.

Revista Iberoamericana, Vol. LXXVIII, Núms. 238-239, Enero-Junio 2012, 259-275
ISSN 2154-4794 (Electrónico) 
aquella validada exclusivamente en el campo de la sociopolítica. ${ }^{9}$ Estemos de acuerdo o no en pensar la ficción utópica como un subgénero de la CF, es evidente que ambos discursos comparten ciertas características, en última instancia aquéllas relacionadas, como señalábamos antes, con la creación de un mundo nuevo. ${ }^{10}$ Ahora bien, resulta interesante señalar aquí que Suvin localiza en esta relación de parentesco una tensión que estaría presente en la mayor parte de la CF moderna. Esta tensión estaría dada por el encuentro de dos fuerzas que encauzarían la escritura en direcciones si no opuestas, al menos divergentes, y que se podrían sintetizar en el narrar y el describir. La ficción utópica, por su interés exclusivo en las construcciones sociopolíticas, privilegiaría la segunda de estas prácticas, a diferencia de la novela individualista, psicológica o simplemente la novela de aventuras. La narrativa de CF, en cambio, se encontraría a mitad de camino entre lo uno y lo otro: narraría, por un la lado, la aventura del protagonista al tiempo que, por el otro, describiría el territorio creado por la ficción. ${ }^{11}$

Está claro que la oposición de estas dos fuerzas se puede experimentar con mayor o menor tensión -pensar la descripción como "lastre" sería un ejemplo de lo primero-, pero en pocas obras adquiere la intensidad que encontramos en Tadeys. El carácter fragmentario de esta "novela” estaría dado en parte por eso, por la presencia de una compulsión a narrar y de un apremio vehemente a diseñar un territorio nuevo, esa voluntad por escribir una novela total que sugería Montaldo. Los múltiples borradores y apuntes sueltos serían el testimonio de esta intensidad, y la forma inacabada que la obra adquiere con ello, paradójicamente, uno de los rasgos que la alejarían del género. En Tadeys, el Estado Espejo de La Comarca es tanto el delirio científico de unos de sus personajes como un universo distópico, y las aventuras que estos llevan a cabo son tanto simples aventuras como los "detalles" que dan espesor al territorio imaginario. Decíamos que el buque-cárcel de amujerar se ofrecía como el espacio utópico de La Comarca; del mismo modo, Tadeys se nos presenta como el espacio utópico del universo literario de Lamborghini, de su escritura extraña.

9 “...utopian fiction is not only, historically, one of the roots of CF, it is also -as argued in chapter 3 of $M O S F$ - logically, if retroactively, one of its forms, that validated by and only by socio-politics. Utopian fiction is the socio-political subgenre of CF, it is social-science-fiction or CF restricted to the field of socio-political relationships or to socio-political constructs understood as crucial for destiny of people." (Suvin, "Science Fiction” 38).

${ }_{10}$ Para un consideración detallada de estas características, ver especialmente las páginas 40-41 de este artículo de Suvin.

${ }^{11}$ Se podría argumentar que toda novela cumple con la tarea de describir su propio territorio ficcional. Sin embargo, en la CF esa descripción juega un papel fundamental, siendo precisamente la primera de sus condiciones de posibilidad.

Revista Iberoamericana, Vol. LXXVIII, Núms. 238-239, Enero-Junio 2012, 259-275
ISSN 2154-4794 (Electrónico) 
UN PUNTO DE VISTA ESTRICTAMENTE CIENTÍFICO

[...] debemos adoptar un punto de vista estrictamente científico. Las cuestiones morales, como ponerse a comparar quiénes son las mejores, si las "se la chupé pensando en otra cosa" o si las que ejercen el derecho de hacer respetar su pudor: tales moralinas está fuera de lugar aquí, puesto que nuestro objetivo es el de identificar el mayor o menor grado de absorción de un modelo, la capacidad de abismar al individuo. (Lamborghini 97)

Este pasaje pertenece también al doctor Emorebe Ky. Se encuentra ya no en su diario, sino en su "pseudo-diario", menos confiable tal vez, como apunta el narrador. Allí se habla de "adoptar un punto de vista estrictamente científico" en la consideración del comportamiento de las "damitas" o "mujercitas", que no son otra cosa que el resultado de la absorción del modelo por parte de los jóvenes violentos.

Lo que me interesa destacar en este momento es precisamente el componente científico presente en Tadeys. Este interés no es casual, dado el empeño del presente artículo. La ciencia es un elemento de indudable centralidad en el género. Si para Suvin el novum era la condición necesaria de la CF, la presencia del saber científico -"the presence of scientific cognition”, dice el crítico- sería la condición suficiente y nos serviría para distinguir esta literatura de otras, como la narrativa fantástica o sobrenatural ("The State of Art” 37). ${ }^{12}$ En definitiva, no habría CF sin la presencia del discurso científico. Afirmar esto último no es banal, aunque pueda parecer evidente y roce la tautología. Tanto más si tenemos en cuenta los modos en que puede aparecer este saber en los textos. Para Suvin no se trata de la mera presencia -a través de hechos e hipótesis- del discurso científico, sino de la utilización de la ciencia como método u horizonte de conocimiento de ese mundo nuevo creado por la obra (38). Es este uso el que contribuiría a determinar este género o esta tendencia que llamamos CF.

Ahora bien, ¿qué papel juega la ciencia en Tadeys?, ¿cómo aparece el discurso científico en el texto más allá de la invocación que hace de él el doctor Ky? Antes de responder a estas preguntas, habría que señalar que la ciencia que encontraremos allí no será una ciencia tecnológica. Esta variante es la que caracteriza quizás a la mayor parte de las obras de CF y la que prima en la versión más popular del género, lo que algunos críticos llamaron hard science fiction. Frente a ella, sin embargo, se levanta otra tradición -la speculative science fiction- en la cual la tecnología y la imaginación científica que la produce están subordinadas a otros elementos que se convierten en el verdadero motor del relato: ya sean las emociones o actitudes

12 "If the novum is the necessary condition of CF (differentiating it from 'naturalistic' fiction), the validation of the novelty by scientifically methodical cognition into which the reader is inexorably led is the sufficient condition for CF" (37).

ISSN 0034-9631 (Impreso) 
humanas, ya sea la política o las ciencias sociales. Angela Dellepiane ha definido, en estos mismos términos, estas dos tendencias en un estudio dedicado precisamente a la narrativa argentina de CF. Y coincidiendo con el grueso de la crítica, ha relacionado la CF argentina e hispanoamericana en general con el segundo de estos dos modelos, la CF especulativa (515). ${ }^{13}$ En este sentido, Tadeys no sería una excepción. Si en la obra aparecen ciertas líneas propias del género, éstas nos conducirían a su versión más soft. El texto de Lamborghini estaría orientada antes a las ciencias sociales - donde el saber psiquiátrico se cruza con la política y las ideas eugenésicas- que al saber tecnológico.

Dicho ésto, debemos precisar ahora cuál es el papel que el discurso científico ocupa en la ficción de Tadeys. En la obra, este saber no se limita a la "mera presencia". Si bien es cierto que el discurso científico del doctor Ky y su puesta en práctica -el barco-cárcel de "amujerar"- no son más que una de las tantas "aventuras” de Tadeys, también es cierto que ambos -discurso y barco- se presentan como la punta de un iceberg. En la medida en que este espacio de confinamiento -definido por un lugar y por un saber- se revela como una miniatura de todo el territorio político que diseña la obra, el discurso científico nos revela su centralidad, es decir, su participación en la construcción y reproducción del Estado y en el diseño de las relaciones sociopolíticas de La Comarca.

Admirablemente, Gabriel Giorgi ha señalado ya este recinto penitenciario como la reinvención del Estado moderno, y lo ha hecho precisamente a través de un fino análisis del discurso del médico alienista: en Tadeys -y no sólo allí- la ciencia médica se revela como el lugar de un saber político sobre el cuerpo; en ella se traza una ecuación entre saber, autoridad y Estado. En este ejercicio de poder sobre los cuerpos individuales y colectivos la ciencia médica se presenta, en definitiva, como los restos de la fundación del Estado moderno, entendiendo este ejercicio de poder como la permanencia de una gestión eugenésica y biopolítica que subyace a pesar de la llegada de otros discursos; su lenguaje -el lenguaje de la ciencia, el lenguaje delirante del doctor Ky- no es otro que el de la ficción normativa (131-40). Tadeys coloca así este rol eminentemente político que adopta el saber científico en el centro de su universo y retoma con ello una tendencia central de la CF. No es sino este uso de la ciencia el que permite pensar esta fábula o ficción utópica, con sus prácticas y técnicas disciplinarias,

${ }^{13}$ Sin entrar necesariamente en la clasificación del género en estas dos vertientes -la cual, como toda clasificación, puede ser en exceso limitante- muchos autores coinciden en esta caracterización de la CF argentina o hispanoamericana. Pablo Capanna, un referente en este campo, señalaría en los años ochenta esto mismo al hablar de los escritores nacionales: "En general, cultivan una literatura fantástica no tradicional, que linda con la ciencia ficción, la atraviesa y sale libremente de su ámbito, con escasa presencia del elemento científico-tecnológico. [...] Quizás el rasgo más común sea que nuestros autores no hacen ciencia ficción a partir de la ciencia, como ocurre en países industriales donde la ciencia impregna la vida diaria; son escritores que se han formado leyendo ciencia ficción y en cuyo mundo espiritual importan las convenciones y los mitos del género" (citado en Souto 19).

Revista Iberoamericana, Vol. LXXVIII, Núms. 238-239, Enero-Junio 2012, 259-275
ISSN 2154-4794 (Electrónico) 
como una reescritura sui generis de obras canónicas dentro del género, tales como $A$ Clockwork Orange (1962), de Anthony Burgess (1917-1993). ${ }^{14}$

¿Pero quiere decir esto que el saber científico en Tadeys funciona de la misma manera que lo hace en el grueso de la CF? Junto con Suvin, habíamos definido el papel de la ciencia en el género como un método u horizonte de conocimiento. Desde esta perspectiva, la CF podría pensarse como un "experimento imaginativo" en el que la ciencia guiaría el relato o la construcción del nuevo territorio ("Science Fiction" 42). Se trataría de un juego: ¿qué pasaría, por ejemplo, si se pudiese condicionar de manera perfecta el comportamiento de los jóvenes violentos? Un resultado posible sería el mundo que Burgess construye en A Clockwork Orange, con sus consecuencias y dilemas morales. La ciencia estaría operando aquí, y en buena parte de las obras del género, como punto de partida y principio rector de la ficción. ${ }^{15}$

Pues bien, en Tadeys la ciencia no ocupa exactamente ese lugar. El discurso científico -sin menoscabar su papel en la definición de La Comarca- se presenta allí antes como una expresión del universo del relato que como su punto de partida. El mecanismo que opera en el condicionamiento de la población y en su transformación en "damitas" de los jóvenes violentos del barco-cárcel, ya lo hemos sugerido, se encuentra también fuera de la ciencia. Se trataría de ese determinismo del cuerpo que recorre buena parte de la obra de Lamborghini, cuya inscripción se encuentra en la propia cultura y, fundamentalmente, en la propia lengua. Por tanto, como veremos a continuación, creo que si tuviéramos que nombrar en Tadeys ese principio rector que encauzara el "experimento imaginativo", no tendríamos que pensar tanto en la ciencia como en la lengua. ¿Qué pasaría si lleváramos al extremo los desvíos de la lengua?, ¿con qué mundo nos encontraríamos?

AMADA LENGUA NATAL

Era una lástima, una traicionera puñalada de la historia, pero gran parte de los giros y vocablos latinos, al pasar a su “amada lengua natal”, se convertían en dobles sentidos, en equívocos tales que ellos, hasta los luchadores contra el fanatismo, advirtieron sinceramente la pezuña del Maligno. (Lamborghini 182)

${ }^{14}$ Robert Scholes y Erik S. Rabkin dedican un pasaje de su obra La ciencia ficción. Historia. Ciencia. Perspectiva a señalar el papel de la psicología, en sus variantes pavloviana y skinneriana, en algunas obras del género. Para estos autores, el condicionamiento mecánico del individuo se convertiría en un importante componente de la CF clásica (168-169).

15 Es evidente que la obra de Burgess excede esta caracterización, pero no es éste el lugar para una estudio más detallado. En esta oportunidad sólo la menciono a guisa de ejemplo de esta manera de pensar el género.

\footnotetext{
Revista Iberoamericana, Vol. LXXVIII, Núms. 238-239, Enero-Junio 2012, 259-275 ISSN 0034-9631 (Impreso) ISSN 2154-4794 (Electrónico)
} 
El gran protagonista de Tadeys -y de toda la obra de Lamborghini- no es otro que la lengua, su "amada lengua natal”. En el pasaje citado arriba, la lengua -en este caso el comarquí- le juega una mala pasada al Padre Maker. Su intento por traducir la Biblia convierte la escritura sagrada en un texto injurioso y soez, "una versión floja de Sade" -como apunta Rosa (120)- llena de "retruécanos obscenos, varios por página” -como insiste Lamborghini (182). Es esta traducción herética la que lo condena al destierro en las montañas del norte, donde descubre a los también obscenos y heréticos tadeys.

Volviendo al género, la lengua ha representado siempre un problema y una oportunidad para la CF (Scholes y Rabkin 171). La invención de un nuevo territorio o la introducción de una nueva raza ha planteado lógicamente el problema del idioma. Si muchos autores han ignorado esta cuestión o utilizado diversos subterfugios para evitar su abordaje, no son pocos los que han asignado al tema un lugar privilegiado en su obra, convirtiéndolo en una oportunidad narrativa más que en una carga. Partiendo del hecho de que cada idioma influye sobre la percepción de la realidad, la caracterización que se haga de aquel ofrece la posibilidad de una nueva reflexión sobre la cultura en cuestión y sobre el lenguaje en general. En su estudio sobre el género, Scholes y Rabkin citan una serie de textos de CF que abordan el problema de la lengua, señalando un crecimiento del interés por la realidad lingüística a partir de los años setenta (172). En la literatura argentina, por mencionar un ejemplo ya citado, el propio Borges ha otorgado a la lengua un lugar central en sus relatos que lindan con el género (“Tlön, Uqbar, Orbis Tertius”, "El informe de Brodie”, etc.). Tadeys confluye en esta línea y se emparenta con el proyecto borgeano en una particularidad que los alejaría en parte de la CF: me atrevería a decir que en ambos autores no es tanto la invención de un nuevo mundo lo que conduce la narración hacia una reflexión sobre el lenguaje como la operación inversa. Es la reflexión sobre la lengua la que parece requerir la construcción de un nuevo territorio o de una nueva raza.

En la obra que nos convoca, la lengua es un elemento omnipresente. La Comarca se define, en primer lugar, a partir de su idioma, el comarquí. Si decíamos que los tadeys, esa raza fantástica de las montañas del norte, estaban llamados a cambiar la fisonomía del país, no es menos cierto que estos seres parecen ser una invención de la propia lengua, una fatalidad del idioma. Porque existe el comarquí, existen los tadeys; de hecho, su descubrimiento por parte del Padre Maker es una de las consecuencias de su "amada lengua natal". ${ }^{16} \mathrm{Y}$ esta amada lengua es abordada en numerosos pasajes del texto: se la describe, en su inestabilidad y en sus deslices, en su permanente doble sentido; se alude a su origen, donde la procedencia latina y eslava se anudan de manera

${ }^{16}$ Quien conozca la obra de Lamborghini sabrá que los tadeys preexisten a Tadeys, lo que podría suponer un argumento en contra de esta última afirmación. Sin embargo, un conocedor tal no podrá negar que el comarquí preexiste a La Comarca.

Revista Iberoamericana, Vol. LXXVIII, Núms. 238-239, Enero-Junio 2012, 259-275
ISSN 2154-4794 (Electrónico) 
inseparable; se postula en ella un secreto inviolable para los extranjeros; se la vuelve objeto de una política de Estado, etc.

Nicolás Rosa ha abordado extensamente la naturaleza del comarquí. En su lectura, la lengua no puede separarse de la política imperial de La Comarca. Es ella la que sostiene el Imperio. Tadeys, en este sentido, estaría poniendo en evidencia el hecho de que las lenguas -como acontecimiento real-histórico y no como "estilo" o "literatura"se convierten generalmente en variantes de una solución fascista (117-118). En efecto, el fantasma de la nominación, del poner nombre a las cosas y la violencia sobre la realidad que supone esta operación recorre el texto, acercándolo así a cierta tendencia del género según la cual los gobiernos autoritarios de los universos distópicos comportarían una política lingüística agresiva. Pero lo curioso aquí -y en la lectura de Rosa- no es tanto el uso político de la lengua como las propias características de este idioma. El comarquí, decíamos, es una lengua inestable. Sus formas son variables. Aunque las autoridades parecen querer limitar sus deslices y su doble sentido, el idioma se resiste -desliz comarquí: la manada se troca en mamada (Lamborghini 216). El juego de palabras se convierte en la norma -"Si sos Tijuán, por el Culo te la dan" (Lamborghini 54)- y el comarquí se vuelve así una lengua desatada, incontrolable. Sus palabras designan siempre otra cosa de lo que deben designar, y la única manera de anclar el sentido en La Comarca pasa por el cuerpo: "En todos los gustos, en todas las variantes, pero siempre, siempre carne y siempre sexual” (Lamborghini 288). Es allí -en el cuerpo de los niños, de los tadeys, de los adolescentes violentos- donde la cadena significante, la cadena del lenguaje, encuentra su límite. Las víctimas -como propone Giorgi- son tanto víctimas de sus verdugos como del lenguaje (126). ${ }^{17}$

Por su destino carnal o sexual -vía asociación fonética, sintáctica o semántica, toda palabra deriva fatalmente en el cuerpo-, el comarquí se presenta como una lengua soez, “[...] una lengua afásica, gutural, libidinosa, que hace grieta en la narración y en la nomenclatura, lengua crasa que desorganiza los paradigmas y al repetirse insiste pornográficamente en algunos, muy pocos, paradigmas sin declinación” (Rosa 12122). Esta lengua, por momentos un juego fácil y banal, adquiere su fuerza debido sobre todo a su insistencia, al hecho de convertirse en la principal aventura de la narración:

${ }^{17}$ Si en toda la obra de Lamborghini el lenguaje tarde o temprano termina actuando sobre los cuerpos es porque en ella el cuerpo se convierte en el origen y el destino de todo lenguaje. La centralidad que tiene la inscripción del género (gender) a través de la lengua en una obra como Tadeys -“El Estado, ¿era hombre o mujer?” se pregunta el narrador (78)- nos recuerda la importancia que adquiere el debate en torno a aquel a la hora de establecer agendas utópicas de distinto cuño. Determinados caminos recorridos por la escritura utópica y la CF reaparecen aquí en la violencia que se ejerce alrededor del género: considerando el género como una configuración donde cuerpo, política y lenguaje se cruzan de manera violenta, se nos abre una perspectiva que si, por un lado, ha sido ya abordada por la crítica -la homosexualidad como uno de los ejes transversales de la obra de Lamborghini-, por el otro, nos sugiere nuevas intersecciones entre Tadeys y la CF.

Revista Iberoamericana, Vol. LXXVIII, Núms. 238-239, Enero-Junio 2012, 259-275
ISSN 2154-4794 (Electrónico) 
"la palabra es consumisión o ardor" nos dice el texto (Lamborghini 290). En la obra, el comarquí no es sólo objeto de estudio o reflexión, sino que trasciende los límites de la ficción y contamina la escritura del relato al punto de que el lector se pregunta si el propio texto que tiene entre manos no está también escrito en esta lengua libidinosa. Como decía, la lengua es el principal protagonista de la ficción. No podía ser de otra manera puesto que -para decirlo con Rosa- el propósito de Tadeys (y de toda la obra de Lamborghini) no es otro que el de "generar una lengua corrupta por dislocación de las formas y de los paradigmas", planteando así un "otro-lenguaje” que en constelación con otros autores cercanos -Perlongher, Zelarrayán- generaría una "literatura-otra" (118).

Este propósito, esta aventura lingüística, excede claramente los límites que la CF asigna a la lengua; y al mismo tiempo la lengua cruza las fronteras de La Comarca. En Tadeys, el idioma se convierte en el nexo entre el territorio imaginario y el más acá. Si, tal como proponen diversos autores, la CF no es sino un desvío para hablar del contexto colectivo del autor, y si esta proposición la arrimáramos a la obra de Lambroghini, habría que decir que el comentario de Tadeys sobre la realidad -el cual podría abarcar áreas diversas- empezaría siempre por la lengua, por su funcionamiento, por su lógica y por los monstruos que engendra su juego y su fatal inscripción en el cuerpo. En este sentido, lo que plantea Tadeys es un comentario sobre nuestra propia lengua, o sobre el lenguaje en general, y por tanto sobre la cultura. Toda lengua es libidinosa, propone el texto. No hace falta más que escuchar. Eso es lo que hace Lamborghini, y eso es lo que hace el Padre Maker. Son los mismos deslices del lenguaje los que destierran al Padre a las montañas del norte y los que confinan a Lamborghini a las cumbres o las profundidades de una literatura-otra.

Su RAREZA

El doctor Ky se proponía investigar una, en fin, rareza de Vich; su rareza, aquella que tal vez fuera la causa de su genio y también su desgracia. [...] Y bien, ahí, encerrado y en sus manos tenían un misterio digno de estudiarse con toda seriedad: el compulsivo onanismo de Vich (trances masturbatorios probablemente acompañados de delirios visionarios, en lenguaje Ky) y su combinación con ingestas tan extremas de alcohol. (Lamborghini 84)

Los personajes de Tadeys son conocidos por sus "rarezas", las cuales -dada la naturaleza del comarquí- no pueden sino ser de orden sexual. Al onanismo compulsivo de Vich -un genio aclamado por la comunidad científica internacional que ha llevado a La Comarca a competir con las grandes potencias de su tiempo- podemos sumar la “rareza sexual” de Rone Vomir. Rone -abuelo de Dam, alcalde omnipotente del paístenía fama de extravagante: amenazaba con la cárcel a las mucamas que trabajaban

Revista Iberoamericana, Vol. LXXVIII, Núms. 238-239, Enero-Junio 2012, 259-275
ISSN 0034-9631 (Impreso) 
en su casa si no le permitían correrse en sus cabelleras en el justo momento en que terminaban de lavarlas. Este tipo de rarezas, como las mismas que descubre el Padre Maker en la cueva de los tadeys, proliferan en el texto, son un elemento constitutivo del territorio imaginario de La Comarca. Allí, junto a las palabras -o a través de ellascircula el goce, y estas rarezas no son otra cosa que el punto donde este goce se afirma en cada individuo.

Estas originalidades de los personajes de La Comarca parecen representar la propia originalidad o la extravagancia de la escritura de Lamborghini, la cual consistiría en permanecer siempre atento a las líneas de goce que vehiculiza la lengua, incluso en los momentos más opresivos. Allí donde el tadey es industrializado y su carne es convertida en mercancía para el comercio sexual, allí reaparece el goce: el tadey goza en su prisión. Allí donde el Estado se empeña en lograr una absorción absoluta del individuo y subsumir su cuerpo a una idea abstracta, desafiando la anatomía humana, allí la narración se encuentra nuevamente con el goce: las “damitas” gozan con sus bufas profesionales. Como sugiere Gabriel Giorgi, ésta es la manera que tiene el texto de afirmar un desvío y una resistencia a ese destino de "sujeto" supuesto en cada cuerpo. Es decir, si el texto plantea, por un lado, el trabajo de la historia sobre los cuerpos, por el otro, no deja de afirmar el goce como un resto y como la reapropiación de dicha carne (124-25). Pero no se trata, cabe señalar, de una reapropiación conciliadora. Ni de un resto "humano" en un mundo "deshumanizado".

En esto, Tadeys parece alejarse definitivamente de ciertas tendencias propias de la CF. En la construcción de universos imaginarios y mundos paralelos, muchos textos del género han querido plasmar los errores o vicios encontrados en su sociedad. Esto le otorgaría a la CF cualidades críticas, pero también didácticas. ${ }^{18}$ La denuncia del presente estaría entre los destinos de esta literatura, al menos en sus formas distópicas -en esta línea y a manera de ejemplo, la ciencia y la técnica se podrían pensar como temas de los que la CF se valdría para concretar novelescamente el problema del hombre atrapado en una civilización tecnocrática que lo oprime a causa, en parte, de un uso desmedido de ambas. La CF, entendida así, se inscribiría en una tendencia moralista de la que se desprendería una mirada no tanto hacia el futuro como hacia el pasado, un pasado perdido y muchas veces idealizado. Como propone Adam Roberts, el modo de la CF no sería tanto el de la profecía como el de la nostalgia (33). ${ }^{19}$

${ }^{18}$ Dellepiane sostiene que es precisamente este "humanismo" lo que habría despertado el interés por el género en Argentina y en otros países de Hispanoamérica (516).

19 En Contra la interpretación, Susan Sontag señalaba esta tendencia bien intencionada en las películas norteamericanas de los años cincuenta: "Las películas de ciencia ficción son marcadamente moralistas. El mensaje característico se refiere al uso adecuado, o humano, de la ciencia contra el uso demente, obsesivo" (255). Y más adelante: "Hay una considerable cantidad de pensamiento bien intencionado en las películas de ciencia ficción, a veces conmovedor, a veces depresivo o deprimente” (259). Es evidente

\footnotetext{
Revista Iberoamericana, Vol. LXXVIII, Núms. 238-239, Enero-Junio 2012, 259-275 ISSN 0034-9631 (Impreso) 
Ahora bien, Tadeys se encuentra muy lejos de estas tendencias del género. Si el principio rector sobre el cual se erige la ficción y todo el territorio de La Comarca es la lengua, los desvíos sexuales y fascistas del comarquí no se presentan allí como un uso abusivo del lenguaje en oposición a un uso más moderado, más humano, sino como formas que están fatalmente inscriptas en la lógica misma de la lengua. Y el goce que se desprende de estas formas, ya lo hemos dicho, dista de "humanizar" el relato. En este aspecto, y en tantos otros, Tadeys no es un texto que se pueda adscribir a la CF. Sin embargo, no se puede negar que estén funcionando allí algunas de sus tendencias. Podríamos decir que, en última instancia, Lamborghini toma lo que encuentra: en Tadeys, la narración utiliza aquellos elementos que son funcionales para su ficción. Esto es lo que quise mostrar a lo largo de este artículo. Esto, y el modo en que -allí donde excede sus líneas directrices- Tadeys arrastra la CF a nuevos territorios.

\section{BiBLIOGRAFÍA}

Barthes, Roland. Sade, Fourier, Loyola. Madrid: Cátedra, 1997.

Borges, Jorge Luis. El informe de Brodie. Buenos Aires: Emecé, 1970.

Burgess, Anthony. A Clockwork Orange.

Dellepiane, Angela B. "Narrativa argentina de ciencia ficción: Tentativas liminares y desarrollo posterior". Actas del IX Congreso de la Asociación Internacional de Hispanistas. Frankfurt am Main: Vervuert Verlag, 1989. 515.

Derrida, Jacques. "The Law of Genre”. Avital Ronell, trad. Critical Inquiry 7/1, (1980): 55-81.

Freedman, Carl. "Science Fiction and Critical Theory". Science Fiction Studies 14/2 (1987): 180-200.

Giménez, Eduardo Abel. “Cuestionario”. La ciencia ficción en la Argentina. Antología crítica. Marcial Souto, compilador. Buenos Aires: Eudeba, 1985. 155.

Giorgi, Gabriel. Sueños de exterminio: homosexualidad y representación en la litertaura argentina contemporánea. Rosario: Beatriz Viterbo, 2004.

Lamborghini, Osvaldo. Tadeys. Buenos Aires: Sudamericana, 2005.

Montaldo, Graciela. "La ficción de las masas". Y todo el resto es literatura. Ensayos sobre Osvaldo Lamborghini. Juan Pablo Dabove y Natalia Brizuela, ed. Buenos Aires: Interzona, 2008. 255-77.

Roberts, Adam. Science Fiction. London: Routledge, 2000.

Rodríguez, Fermín. "Escribir afuera: literatura y política en Walsh y Lamborghini. (Para una lectura de Tadeys)". Y todo el resto es literatura. Ensayos sobre Osvaldo

que esta caracterización no puede hacerse extensiva a todo el género -ni en la literatura ni en el cine-, pero subsiste -con mayor o menor intensidad- en buena parte de sus obras.

$\begin{array}{llll}\text { ISSN 0034-9631 (Impreso) } & \text { ISSN 2154-4794 (Electrónico) }\end{array}$ 
Lamborghini. Juan Pablo Dabove y Natalia Brizuela, eds. Buenos Aires: Interzona, 2008. 155-81.

Rosa, Nicolás. Usos de la literatura. Valencia: Grup d'Estudis Iberoamericans y Tirant lo Blanch, 1999.

Scholes, Robert y Eric S. Rabkin. La ciencia ficción. Historia. Ciencia. Perspectiva. Madrid: Taurus, 1982.

Sontag, Susan. Contra la interpretación. Barcelona: Seix Barral, 1969.

Souto, Marcial, comp. La ciencia ficción en la Argentina. Antología crítica. Buenos Aires: Eudeba, 1985.

Strafacce, Ricardo. Osvaldo Lamborghini, una biografía. Buenos Aires: Mansalva, 2008.

Suvin, Darko. "Science Fiction and Utopian Fiction: Degrees of Kinship”. Positions and Presuppositions in Science Fiction. Kent: Kent State UP, 1998. 33-43.

"The State of the Art in Science Fiction Theory: Determining and Delimiting the Genre”. Science Fiction Studies 6/1 (1979): 32-45.

Revista Iberoamericana, Vol. LXXVIII, Núms. 238-239, Enero-Junio 2012, 259-275
ISSN 0034-9631 (Impreso) 
